\title{
La Transformada de Fourier para explicar el proceso de Tomografía Computarizada
}

\author{
Edwin Chávez Ramírez ${ }^{1}$, Efraín Carbajal Peña ${ }^{2}$, \\ Victoriano Yauri Luque ${ }^{3}$, Cristian Amador Loli Prudencio ${ }^{4}$
}

Resumen: La tomografía computarizada (TC) es un gran avance tecnológico utilizado en medicina para diagnósticar enfermedades. La misma técnica es usada en otras áreas, tales como química aeroespacial, radioastronomia, etc. El principal objetivo de la TC es reconstruir la parte interna de un objeto sin tener la necesidad de abrirlo, dicho procedimiento es conocido como un ensayo no destructivo. Para lograr este propósito, la TC usa la atenución que sufren los rayos $X$ al atravesar por el objeto con dicha información, se reconstruye la parte interna del objeto, es decir la TC se trata de un problema inverso.

En este artículo se utiliza con la transformada de Fourier para la reconstrucción de la parte interna, para ello se parte directamente de un modelo continuo, representando la densidad en el punto $(x, y)$ de una sección considerada, con una función $\rho=\rho(x, y)$; y aunque no se requiera que $\rho$ sea continua, el método de Fourier es más eficiente y se conseguirá un mejor resultado cuanto más regular sea $\rho$.

Palabras clave: Tomografía computarizada; transformada de Fourier; problema inverso.

\section{Fourier Transform To Explain The Process Of Computed Tomography}

\begin{abstract}
Computed tomography $(\mathrm{CT})$ is a breakthrough technology used in medicine for diagnostic diseases. The same technique is used in other areas, such as aerospace chemistry, Radio astronomy, etc. The main objective of the $\mathrm{CT}$ is to reconstruct the internal part of an object without having to open it as a non-destructive test. To achieve this purpose, the CT uses the attention that X-rays undergo through this object with the information, the inner part of the object is reconstructed, that is to say the $\mathrm{CT}$ is an inverse problem.

In this article uses the Fourier transform for the reconstruction of the internal part, for it is directly part of a continuous model, representing the density at the point $(x, y)$ of a section, with a function $\rho=\rho(x, y)$; and even though $\rho$ is not required to be continuous, the Fourier method is more efficient and a better, more regular result is obtained $\rho$.
\end{abstract}

Keywords: Computed tomography; Fourier transformation; inverse problem

Recibido: 14/04/2017. Aceptado: 07/07/2017. Publicado online: 01/09/2017

(C)Los autores. Este artículo es publicado por la Revista PESQUIMAT de la Facultad de Ciencias Matemáticas, Universidad Nacional Mayor de San Marcos. Este es un artículo de acceso abierto, distribuido bajo los términos de la licencia Creative Commons Atribucion-No Comercia-Compartir Igual 4.0 que la obra original sea debidamente citada. Para información, por favor póngase en contacto con revistapesquimat.matematica@unmsm.edu.pe

${ }^{1}$ UNMSM, Facultad de Ciencias Matemáticas. e-mail: edwin.chavez@unmsm.edu.pe

${ }^{2}$ UNMSM, Facultad de Ciencias Matemáticas, e-mail:ecarpe@unmsm.edu.pe

${ }^{3}$ UNMSM, Facultad de Ciencias Matemáticas, e-mail:vyauril@unmsm.edu.pe

${ }^{4}$ UNMSM, Facultad de Ciencias Matemáticas, e-mail: clolip@unmsm.edu.pe 


\section{Introducción}

Desde la década de los 70 del siglo pasado se han desarrollado diversos métodos para poder visualizar el interior de las personas de forma virtual (ver [10]), lo cual es de gran utilidad en la práctica médica. En este artículo, se revisa el método que utiliza la transformada de Fourier (ver [4]), para la tomografía computarizada. El método de la reconstrucción algebraica vista, a pesar de su sencillez, no es del todo satisfactoria por su lentitud e imprecisión ([8]). Parte de esta imprecisión se debe a que desde el inicio, se discretiza usando una malla que simula los cambios continuos en la densidad, sólo cuando el sistema lineal asociado tiene dimensiones gigantescas [3].

Para evitar ese inconveniente, se parte directamente del modelo continuo, sin modificar la idea de representar con una función $\rho=\rho(x, y)$ la densidad en el punto $(x, y)$ de la sección considerada. Por cierto, aunque no se requiera que $\rho$ sea continua, este método será más eficiente cuanto más regular sea $\rho,[3]$.

La tomografía computarizada (TC) utiliza la atenuación ${ }^{1}$ de la intensidad que sufren los rayos $X$ al atravesar por los tejidos, lo que nos lleva a introducir un pequeño modelo,

$$
\ln \left(\frac{I_{0}}{I}\right)=\int_{L} \rho(x, y) d L
$$

el cual es una versión simplificada de la llamada ecuación del transporte, los detalles los dan A. Ramm et al.[12, pág. 6], donde $\rho$ es la densidad del objeto y $I, I_{0}$ son las intensidades final e inicial respectivamente.

Supongase que atraviese la muestra con un haz paralelo de rayos $X$ que se proyectan ortogonalmente sobre una recta exterior que forma un ángulo $\theta$ con el eje $O X$, como se ilustra en la figura 1.

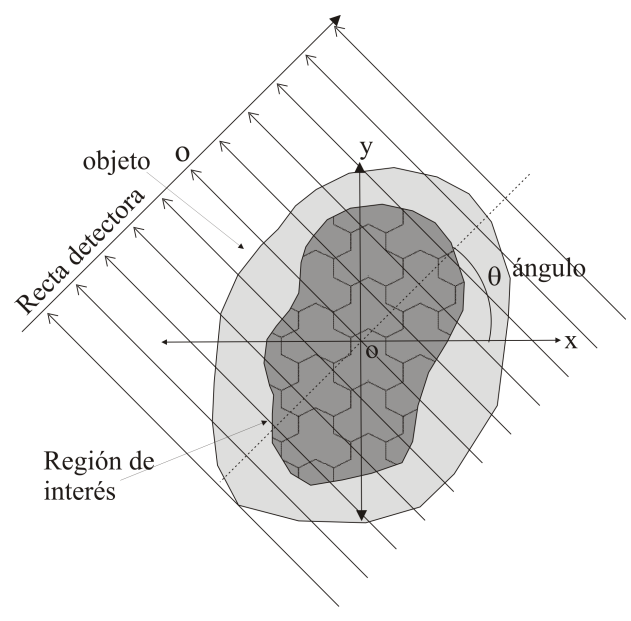

Figura 1: Muestra atravesada por rayos paralelos. Fuente. Chávez (2009, p. 48)

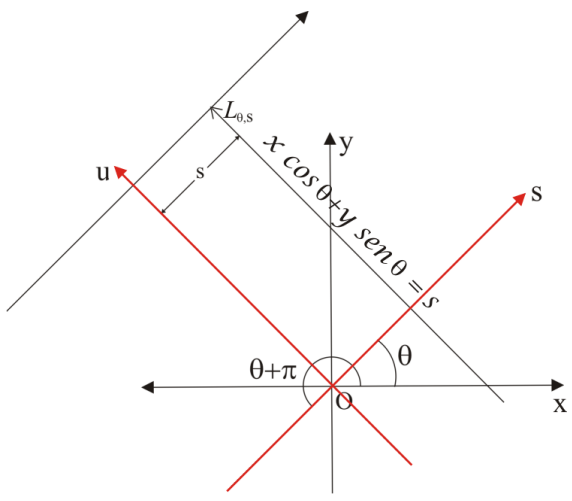

Figura 2: El rayo $L_{(\theta, s)}$ y los dos sistemas. Fuente. Chávez (2009, p. 49)

Esta recta (recta detectora de la figura 1) se puede identificar con la recta real $\mathbb{R}$ y situar el origen en el punto de intersección con el rayo que pasa por $(0 ; 0)$, y obtener un nuevo sistema de coordenadas el cual se denotará por $(s ; u)$, como se muestra en la figura 2 .

Un simple dibujo muestra que el rayo $L_{\theta ; s}$ que pasa por el punto $s$ de esta recta tiene ecuación $L_{\theta ; s}=$ $x \cos \theta+y \operatorname{sen} \theta-s=0$. Como la atenuación que ha experimentado el rayo cuando llega a $s$ dependerá de la cantidad de masa que haya atravesado, es decir, de la integral de línea: (para detalles ver [3], [4] [8])

$$
P_{\theta}(s)=\int_{L_{\theta, s}} \rho .
$$

\footnotetext{
${ }^{1}$ se denomina atenuación de una señal, sea esta acústica, eléctrica u óptica, a la pérdida de potencia sufrida por la misma al transitar por cualquier medio de transmisión.
} 
Notando que $L_{\theta, s}$ y $L_{\theta+\pi, s}$ son rectas idénticas o simplemente imaginando la muestra rodeada de rectas detectoras con orientación compatible, se tiene $P_{\theta}(s)=P_{\theta+\pi}(-s)$.

El operador que asigna a una función escalar su integral de línea sobre cada recta es esencialmente lo que se llama transformada de Radon o, por razones obvias, transformada de rayos $X$. Para cada $\theta$, la función $P_{\theta}(s)$ indica la "sombra" de la muestra, que es translúcida a los rayos $X$, sobre una pared que forma ángulo $\theta$ con la horizontal. (Ver la sección 1.2 de [4])

El problema de calcular la transformada de Radon $(\mathcal{R} f$ ) cuando se conoce el valor de $f$, es conocido como problema directo, mientras que determinar la densidad $f$ cuando se conoce la transformada de Radon $\mathcal{R} f$ es conocido como problema inverso, más detalles se pueden ver en [2], [4], [7], [9].

En 1917 J. Radon consiguió una fórmula (ver [13]) que permite obtener la función original $\rho$ a partir de todas sus sombras $P_{\theta}(s)$, lo cual tiene algunas consecuencias en las Matemáticas puras (por ejemplo permite deducir una fórmula de D'Alambert para la ecuación de ondas en $\mathbb{R}^{3}$, ver [5]). Sin embargo, tuvieron que pasar aproximadamente 60 años para que se convirtiera en un tema fundamental de las Matemáticas aplicadas.

\section{Preliminares}

Parametrizar un rayo que tenga una distancia $s$ al origen de coordenadas y un ángulo $\theta$, como en la figura 2 , entonces se tiene que la recta $L_{(\theta, s)}$ está parametrizada por

$$
\left\{\begin{array}{l}
x=s \cos \theta-u \operatorname{sen} \theta \\
y=s \operatorname{sen} \theta+u \cos \theta ; \text { para } u \in \mathbb{R} .
\end{array}\right.
$$

Con esta parametrización se puede reescribir la ecuación (1)

$$
\ln \left(\frac{I_{0}}{I}\right)=\int_{L} \rho(x, y) d L=\int_{-\infty}^{\infty} f(s \cos \theta-u \operatorname{sen} \theta, s \operatorname{sen} \theta+u \cos \theta) d u .
$$

Ahora aprovechando la figura 2 se define una rotación que será usada más adelante en la demostración del Fourier Slice Theorem $T: \mathbb{R}^{2} \rightarrow \mathbb{R}^{2}$ que lleva las coordenadas $(s, u)$ en el sistema $(x, y)$ definida como:

$$
T:\left\{\begin{array}{l}
x=s \cos \theta-u \operatorname{sen} \theta \\
y=s \operatorname{sen} \theta+u \cos \theta
\end{array}\right.
$$

Esta transformación es invertible y su inversa $T^{-1}: \mathbb{R}^{2} \rightarrow \mathbb{R}^{2}$ está dada por:

$$
T^{-1}:\left\{\begin{array}{l}
s=x \cos \theta+y \operatorname{sen} \theta \\
u=-x \operatorname{sen} \theta+y \cos \theta
\end{array}\right.
$$

Como se comentó, la atenuación que experimenta el rayo cuando llega a $s$ dependerá de la cantidad de masa que haya atravesado, es decir, la atenuación dependerá de la integral de línea, o la proyección paralela (ver $[4])$.

$$
\mathcal{P}_{\theta} f(s)=\int_{L_{(\theta, s)}} f(x, y) d L .
$$

Luego, con la ecuación (6) y la parametrización de la ecuación (2) se puede escribir

$$
\mathcal{P}_{\theta} f(s)=\int_{L_{(\theta, s)}} f(x, y) d L=\int_{-\infty}^{\infty} f(s \cos \theta-u \operatorname{sen} \theta, s \operatorname{sen} \theta+u \cos \theta) d u .
$$

Notando que $L_{(\theta, s)}$ y $L_{(\theta+\pi,-s)}$ son rectas idénticas ${ }^{2}$ o simplemente imaginando la muestra rodeada de rectas detectoras con orientación compatible, se tiene $\mathcal{P}_{\theta} f(s)=\mathcal{P}_{\theta+\pi} f(-s)$.

Matemáticamente se enfrenta el problema de tener que hallar una función conociendo sus integrales de línea

\footnotetext{
${ }^{2}$ Simetría del haz $\Rightarrow L_{(\theta, s)}=L_{(\theta+\pi,-s)}$
} 
en las distintas direcciones. Es decir, lo que se busca es una fórmula, como la de Radon, que nos facilite encontrar $f$ a partir de las proyecciones $\mathcal{P}_{\theta} f(s)$. Hay varias posiblidades las cuales llevan a varias fórmulas equivalentes, ver por ejemplo [10]. Se ve una fórmula conocida como teorema de las rebanadas de Fourier en el campo tomográfico.

El Teorema de las rebanadas de Fourier es una generalización de un hecho muy sencillo:

La fórmula del teorema de las rebanadas se encuentra tomando la transformada de Fourier en una dimensión de una proyección paralela y notando que es igual a una rebanada de la transformada de Fourier en dos dimensiones del objeto original.

Comenzamos definiendo la transformada de Fourier y su transformada inversa en una y en dos dimensiones de la función $f(\xi)$ y de $f(x, y)$ respectivamente:

Transformada de Fourier en una dimensión

$$
\mathcal{F} f(\xi)=\widehat{f}(\xi)=\int_{-\infty}^{\infty} f(x) e^{-2 \pi i x \xi} d x .
$$

Transformada de Fourier en dos dimensiones

$$
\mathcal{F} f(s, u)=\widehat{f}(s, u)=\int_{-\infty}^{\infty} \int_{-\infty}^{\infty} f(x, y) e^{-2 \pi i(s x+u y)} d x d y .
$$

Transformada de Fourier inversa en una dimensión

$$
f(x)=\mathcal{F}^{-1} \widehat{f}(x)=\int_{-\infty}^{\infty} \widehat{f}(\xi) e^{2 \pi i x \xi} \xi .
$$

Transformada de Fourier inversa en dos dimensiones

$$
f(x, y)=\mathcal{F}^{-1} \widehat{f}(x, y)=\int_{-\infty}^{\infty} \int_{-\infty}^{\infty} \widehat{f}(s, u) e^{2 \pi i(s x+u y)} d s d u .
$$

Luego, si se considera $\theta=0^{\circ}$ en la ecuación de la proyección (7), entonces $\mathcal{P}_{0} f(s)$ no es más que la integral sobre la recta vertical $x=s$.

$$
\mathcal{P}_{0} f(s)=\int_{-\infty}^{\infty} f(s \cos 0-u \operatorname{sen} 0, s \operatorname{sen} 0+u \cos 0) d u=\int_{-\infty}^{\infty} f(s, u) d u .
$$

Por otro lado tomando la transformada de Fourier bidimensional en el caso particular usando $u=0$ se obtiene

$$
\begin{aligned}
\widehat{f}(s, 0)=\mathcal{F} f(s, 0) & =\int_{-\infty}^{\infty} \int_{-\infty}^{\infty} f(x, y) e^{-2 \pi i(s x+0 y)} d y d x=\int_{-\infty}^{\infty} \int_{-\infty}^{\infty} f(x, y) e^{-2 \pi i(s x)} d y d x \\
& =\int_{-\infty}^{\infty}\left[\int_{-\infty}^{\infty} f(x, y) d y\right] e^{-2 \pi i s x} d x=\int_{-\infty}^{\infty} \mathcal{P}_{0} f(x) e^{-2 \pi i s x} d x=\widehat{\mathcal{P}_{0} f}(s) .
\end{aligned}
$$

Por lo tanto,

$$
\widehat{f}(s, 0)=\widehat{\mathcal{P}_{0} f}(s) .
$$

Es decir que la transformada de Fourier bidimensional de $f$ evaluada en el eje $X$ se puede hallar integrando $f$ en la recta vertical $L_{(0, s)}$ y después calculando la transformada de Fourier unidimensional de la función resultante, finalmente si queremos despejar $f$ bastará tomar transformadas inversas.

Este hecho puede ser extendido al caso general de modo que $\widehat{f}(s, \theta)$ evaluada en una recta que forma un ángulo $\theta$ con el eje $X$ y que pasa por el origen debe coincidir siempre con $\widehat{\mathcal{P}_{\theta} f}(s)$ y luego tomando transformadas inversas podemos despejar $f$.

Por otro lado, desde el punto de vista matemático, uno ya podría estar satisfecho, sin embargo sigue la pregunta y cómo podemos resolver esto numéricamente, pues la computadora no reconoce a las integrales ni a las proyecciones, veamos las siguiente fórmulas las cuales nos permitiran poner las formulas anteriores en 
un programa computacional, los detalles se pueden encontrar en [4, Apéndice A3].

Los coeficientes de Fourier de una función par $f(x)$ se pueden obtener, de manera simplificada, mediante las siguientes fórmulas:

$$
a_{n}=\frac{2}{l} \int_{0}^{l} f(x) \cos \left(\frac{n \pi x}{l}\right) d x \quad \mathrm{y} \quad b_{n}=0 .
$$

Por consiguiente, la serie de Fourier de una función par contiene solo los cosenos, es decir, tiene la forma:

$$
f(x)=\frac{1}{2} a_{0}+\sum_{n=1}^{\infty} a_{n} \cos \left(\frac{n \pi x}{l}\right) .
$$

\section{Teorema Principal:Teorema de las rebanadas o el Fourier Slice Theorem}

La dificultad matemática a la que nos enfrentamos es hallar una función conociendo sus integrales de línea en todas las direcciones, es decir, lo que busca es una fórmula, que permita recuperar $\rho$ a partir de las funciones $\mathcal{P}_{\theta}(t)$.

Hay algunas fórmulas equivalentes para este objetivo en [8, sección 2.2]. En la presente sección se ve una fórmula que es conocida como Fourier Slice Theorem (teorema de las rebanadas de Fourier).

Teorema 3.1 (Fourier Slice Theorem)

Sea $\mathcal{P}_{\theta} f(s)$ la integral de línea de $f$ sobre la recta $L_{(\theta, s)}$ con la parametrización dada en la ecuación (2), entonces para $f$ suficientemente regular se tiene

$$
f(x, y)=\int_{0}^{\pi} \int_{-\infty}^{\infty}|r| \widehat{\mathcal{P}_{\theta} f}(r) e^{2 \pi i(x r \cos \theta+y r \operatorname{sen} \theta)} d r d \theta
$$

Demostración. Primero veamos que $\widehat{\mathcal{P}_{\theta} f}(r)=\widehat{\mathcal{P}_{\theta+\pi} f}(-r)$, en efecto, con la ayuda de la figura 3 se obtiene que $(r, \theta)=(-r, \theta+\pi)$ como los puntos son iguales entonces y por la simetría del haz, las rectas en esos puntos son iguales, es decir, $L_{(\theta, s)}=L_{(\theta+\pi, s)}$, luego las correspondientes proyecciones serán iguales, entonces $\mathcal{P}_{\theta} f(r)=\mathcal{P}_{\theta+\pi} f(-r)$. Si tomamos la transformada de Fourier tenemos $\widehat{\mathcal{P}_{\theta} f}(r)=\widehat{\mathcal{P}_{\theta+\pi} f}(-r)$ que era lo que se estaba buscando.

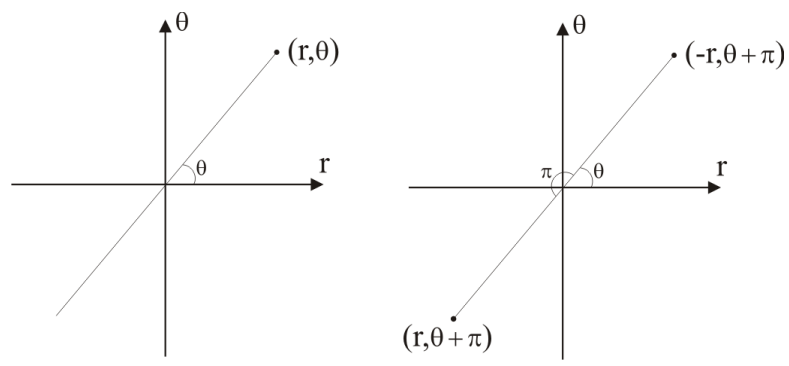

Figura 3: Simetría del haz.

Fuente. Chávez (2009, p. 64)

Si en la ecuación (15) se separa los límites de integración de menos infinito a cero y de cero a infinito y se hace un cambio de variable en el primer término, <se obtiene

$$
\begin{aligned}
f(x, y)= & \int_{0}^{\pi}\left[\int_{\infty}^{0} r \widehat{\mathcal{P}_{\theta} f}(-r) e^{2 \pi i(x(-r) \cos \theta+y(-r) \operatorname{sen} \theta)}-d r\right] d \theta \\
& +\int_{0}^{\pi}\left[\int_{0}^{\infty} r \widehat{\mathcal{P}_{\theta} f}(r) e^{2 \pi i(x r \cos \theta+y r \operatorname{sen} \theta)} d r\right] d \theta .
\end{aligned}
$$

PESQUIMAT 20(1): 77-92 
Cambiando los límites de integración en el primer termino y usando el hecho que $\widehat{\mathcal{P}_{\theta} f}(-r)=\widehat{\mathcal{P}_{\theta+\pi} f}(r)$ y haciendo $\alpha=\theta+\pi$, se tiene

$$
\begin{aligned}
f(x, y)=\int_{\pi}^{2 \pi} & {\left[\int_{0}^{\infty} r \widehat{\mathcal{P}_{\alpha} f}(r) e^{-2 \pi i(-x r \cos \alpha-y r \operatorname{sen} \alpha)} d r\right] d \alpha } \\
+ & \int_{0}^{\pi}\left[\int_{0}^{\infty} r \widehat{\mathcal{P}_{\theta} f}(r) e^{2 \pi i(x r \cos \theta+y r \operatorname{sen} \theta)} d r\right] d \theta .
\end{aligned}
$$

Regresando a la variable $\theta$ y juntando las integrales, se llega a:

$$
f(x, y)=\int_{0}^{2 \pi} \int_{0}^{\infty} r \widehat{\mathcal{P}_{\theta} f}(r) e^{2 \pi i(x r \cos \theta+y r \operatorname{sen} \theta)} d r d \theta .
$$

Por otro lado, usando la inversión de la transformada de Fourier ecuación (11)

$$
f(x, y)=\int_{-\infty}^{\infty} \int_{-\infty}^{\infty} \widehat{f}(s, u) e^{2 \pi i(s x+u y)} d x d y
$$

Haciendo el cambio de variables $(s, u)$ a coordenadas polares $s=r \cos \theta$ y $u=r \operatorname{sen} \theta$; se obtiene

$$
f(x, y)=\int_{0}^{2 \pi} \int_{0}^{\infty} r \widehat{f}(r \cos \theta, r \operatorname{sen} \theta) e^{2 \pi i(x r \cos \theta+y \operatorname{sen} \theta)} d r d \theta .
$$

Comparando las ecuaciones (16) y (17), entonces basta probar que

$$
\widehat{\mathcal{P}_{\theta} f}(r)=\widehat{f}(r \cos \theta, r \operatorname{sen} \theta) .
$$

Para ello consideramos una rotación de ángulo $\theta$ alrededor del origen, y llegamos a la transformación dadas en (4).

$$
T:\left\{\begin{array}{l}
x=s \cos \theta-u \operatorname{sen} \theta \\
y=s \operatorname{sen} \theta+u \cos \theta
\end{array}\right.
$$

Por otro lado, la ecuación (7), también puede ser escrita como composición de $f$ con $T$

$$
\begin{aligned}
\mathcal{P}_{\theta} f(s) & =\int_{-\infty}^{\infty} f(s \cos \theta-u \operatorname{sen} \theta, s \operatorname{sen} \theta+u \cos \theta) d u \\
& =\int_{-\infty}^{\infty} f(T(s, u)) d u=\int_{-\infty}^{\infty}(f \circ T)(s, u) d u .
\end{aligned}
$$

Por lo tanto,

$$
\mathcal{P}_{\theta} f(s)=\int_{-\infty}^{\infty}(f \circ T)(s, u) d u
$$

Luego, aplicando la definición de transformada de Fourier unidimensional a $\mathcal{P}_{\theta} f(r)$ y usando la ecuación (18) se tiene

$$
\widehat{\mathcal{P}_{\theta} f}(r)=\int_{-\infty}^{\infty} \mathcal{P}_{\theta} f(s) e^{-2 \pi i r s} d s=\int_{-\infty}^{\infty}\left[\int_{-\infty}^{\infty}(f \circ T)(s, u) d u\right] e^{-2 \pi i r s} d s
$$

Luego

$$
\widehat{\mathcal{P}_{\theta} f}(r)=\int_{-\infty}^{\infty} \int_{-\infty}^{\infty}(f \circ T)(s, u) e^{-2 \pi i r s} d u d s .
$$

Finalmente, se hace el hacer el cambio de variables dado en la ecuación (5) para la ecuación (19). El cambio está dado por

$$
T^{-1}: \begin{cases}s= & x \cos \theta+y \operatorname{sen} \theta, \\ u= & -x \operatorname{sen} \theta+y \cos \theta,\end{cases}
$$


como determinante del jacobiano $|J(s, u)|=1$, entonces se obtiene

$$
\begin{aligned}
\widehat{\mathcal{P}_{\theta} f}(r) & =\int_{-\infty}^{\infty} \int_{-\infty}^{\infty}(f \circ T)(s, u) e^{-2 \pi i r s} d u d s \\
& =\int_{-\infty}^{\infty} \int_{-\infty}^{\infty}(f \circ T)\left(T^{-1}(x, y)\right) e^{-2 \pi i r(x \cos \theta+y \operatorname{sen} \theta)} d u d s \\
& =\int_{-\infty}^{\infty} \int_{-\infty}^{\infty} f(x, y) e^{-2 \pi i(x(r \cos \theta)+y(r \operatorname{sen} \theta))} d y d x .
\end{aligned}
$$

Por lo tanto:

$$
\widehat{\mathcal{P}_{\theta} f}(r)=\widehat{f}(r \cos \theta, r \operatorname{sen} \theta)
$$

Con lo que se concluye la demostración.

Observación 1. Cuando el teorema 3.1 exige que $f$ sea suficientemente regular, se exige esto para poder aplicar la fórmula de inversión, y cuando se tienen funciones de soporte compacto integrables y acotadas, es posible tener igualdades en casi todo punto. De este modo se puede satisfacer lo requerido desde el punto de vista teórico. En el campo práctico no se va a desistir de ver un tumor o cualquier objeto que tenga grosor porque $f$ no sea $C^{\infty}$. Sin embargo, se debe tener presente que la falta de regularidad aunada a los métodos aproximados que se emplean, podría crear sombras que en la realidad no existen.

Se puede ilustrar este hecho mediante la figura 4, en la que se ilustra el Teorema de las rebanadas:

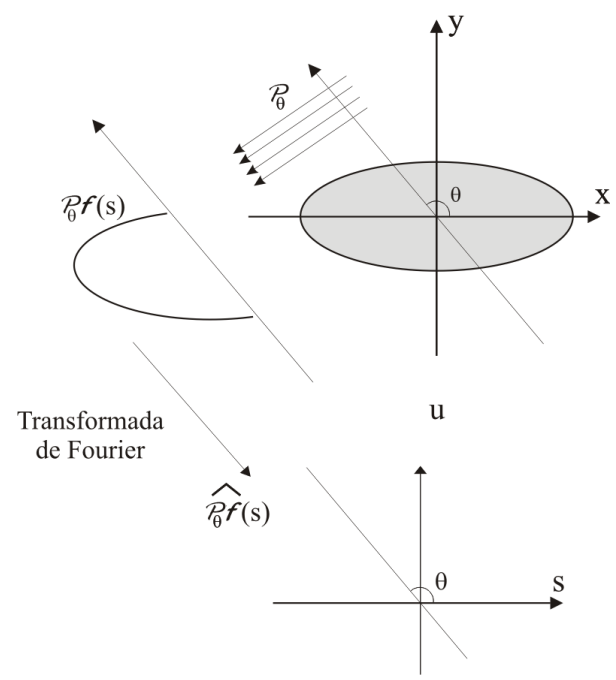

Figura 4: El Teorema de las rebanadas.

Fuente. Chávez (2009, p. 63)

La Transformada de Fourier unidimensional de una proyección paralela formando un ángulo $\theta$ con el eje $X$ es igual a los valores de la transformada bidimensional de un objeto a lo largo de una línea recta que pasa por el origen, formando un mismo ángulo $\theta$ con el eje $s$.

La ecuación (15) del teorema 3.1, es la ecuación principal del método de la retroproyección filtrada (backprojected). Este nombre surge de la interpretación que se le da, pues la ecuación (15) puede ser dividida en dos integrales, es decir, se puede reescribir como:

$$
f(x, y)=\int_{0}^{\pi} Q_{\theta}(x \cos \theta+y \operatorname{sen} \theta) d \theta,
$$


donde

$$
Q_{\theta}(s)=\int_{-\infty}^{\infty}|r| \widehat{\mathcal{P}_{\theta} f}(r) e^{2 \pi i r s} d r
$$

La ecuación (22) es la transformada inversa de Fourier del producto de $|r| \mathcal{P}_{\theta} f(r)$; esta ecuación representa un filtrado de proyección, donde la frecuencia responsable del filtro es dado por $|r|$. (Tomado de [8]). $Q_{\theta}(s)$ es llamada proyección filtrada (filtered proyection), este filtro es conocido como filtro rampa (está siendo representada en la figura 5), o filtro de Ramachandran-Lakshminarayanan o simplemente filtro de Ram-Lak.

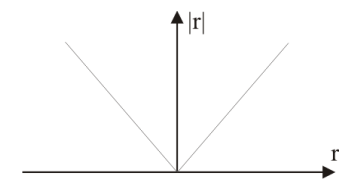

Figura 5: Filtro Rampa.

Fuente. Chávez (2009, p. 67)

El valor de un determinado punto de coordenadas $(x, y)$ de la imagen original es dado por la sumatoria (o integral) de los diversos valores de $Q_{\theta}(x \cos \theta+y \operatorname{sen} \theta)$ para varios valores de $\theta$ entre 0 a $\pi$. La figura 6 , muestra el significado de la contribución de la proyección filtrada para cualquier $Q_{\theta_{1}}$.

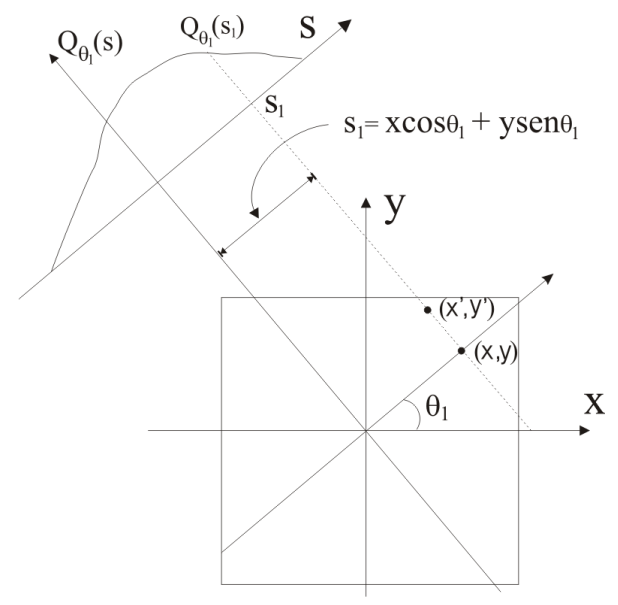

Figura 6: Retroproyección para la reconstrucción de una imagen.

Fuente. Chávez (2009, p. 67)

Para el ángulo $\theta_{1}$, el valor de la proyección filtrada $Q_{\theta_{1}}(s)$ que contribuirá para el valor final en el punto $(x, y)$ es dado por la coordenada $s_{1}=x \cos \theta_{1}+y \operatorname{sen} \theta_{1}$. Para el mismo ángulo $\theta_{1}$ en el punto $\left(x^{\prime}, y^{\prime}\right)$, el valor de la coordenada en el eje $s$ será el mismo: $s_{1}^{\prime}=x^{\prime} \cos \theta_{1}+y^{\prime} \operatorname{sen} \theta_{1}=s_{1}$.

Por consiguiente, la proyección filtrada $Q_{\theta_{1}}\left(s_{1}\right)$, hará la misma contribución para la reconstrucción de todos estos puntos en la región de la imagen original, contribuyendo para todos los puntos a lo largo de la reta $s=s_{1}$. Todos los valores de $Q_{\theta_{1}}(s)$ son de esta manera retroproyectados en la región de la imagen original. Este proceso es realizado para todos los ángulos $\theta_{i}$ en que fueran adquiridas las proyecciones $\mathcal{P}_{\theta_{i}} f(s)$.

Una vez conseguida una fórmula exacta que resuelve el problema matemático surge la pregunta: ¿y ahora cómo se introduce esta fórmula en una computadora?; entonces el trabajo aún no ha terminado. 


\subsection{Tratamiento Numérico}

El método natural para tratar numéricamente expresiones que involucren integrales o series de Fourier es la transformada de Fourier discreta y la transformada de Fourier rápida (para más detalle ver [14]) más conocida por sus siglas en inglés $F F T$. Este método, junto con el desarrollo de las computadoras, ha cambiado muchos métodos numéricos desde su introducción en ls década de 1960.Aquíse menciona una versión compacta de la que se muestra en [8].

De las ecuaciones (15) y (22) se obtiene

$$
f(x, y)=\int_{0}^{\pi} Q_{\theta}(x \cos \theta+y \operatorname{sen} \theta) d \theta
$$

donde

$$
Q_{\theta}(s)=\int_{-\infty}^{\infty}|r| \widehat{\mathcal{P}_{\theta} f}(r) e^{2 \pi i r s} d r
$$

Donde se puede ver que el problema se reduce a saber aproximar $Q_{\theta}(s)$, y podemos usar ([1]) para buscar un método(regla del trapecio, de Simpson, cuadratura de Gauss,etc.) que nos ayude a aproximar integrales sobre el intervalo $[0, \pi]$.

Entonces el objetivo será aproximar la función $Q_{\theta}(s)$ para ello se usará las series de Fourier.

Desarrollese la función $f(x)=|x|$ por series de Fourier en el intervalo [-1/2,1/2], para ello se usará fórmulas ${ }^{3}$ (ver [4]), para hallar $a_{0}, a_{n}$ y $b_{n}$, además teniendo en cuenta que $f(x)=|x|$ es una función par, por la ecuación (14) se tiene que $b_{n}=0$. Ahora solo falta hallar $a_{0}$ y $a_{n}$

$$
a_{0}=2 \int_{-\frac{1}{2}}^{\frac{1}{2}}|x| \cos (2 * 0 * \pi x) d x=4 \int_{0}^{\frac{1}{2}} x d x=\frac{1}{2} .
$$

Hallando $a_{n}$

$$
a_{n}=2 \int_{-\frac{1}{2}}^{\frac{1}{2}}|x| \cos (2 n \pi x) d x=4 \int_{0}^{\frac{1}{2}} x \cos (2 n \pi x) d x=\frac{(-1)^{n}-1}{n^{2} \pi^{2}} .
$$

Luego, se tiene

$$
|x|=\frac{a_{0}}{2}+\sum_{n=1}^{\infty}\left(a_{n} \cos (2 n \pi x)\right) \quad \text { con } \quad a_{0}=\frac{1}{2}, a_{n}=\frac{(-1)^{n}-1}{n^{2} \pi^{2}} .
$$

Recordando que

$$
\cos (2 n \pi x)=\frac{e^{2 n \pi i x}+e^{-2 n \pi i x}}{2} .
$$

Entonces, la ecuación (25), se puede reescribir como:

$$
\begin{aligned}
|x| & =\frac{1}{4}+\frac{1}{2} \sum_{n=1}^{\infty} a_{n}\left(e^{2 n \pi i x}+e^{-2 n \pi i x}\right) \\
& =\frac{1}{4}+\frac{1}{2} \sum_{n=1}^{\infty} a_{n}\left(e^{2 n \pi i x}\right)+\frac{1}{2} \sum_{n=1}^{\infty} a_{n}\left(e^{-2 n \pi i x}\right) .
\end{aligned}
$$

Si se toma $m=-n$, en la última sumatoria se tiene

$$
|x|=\frac{1}{4}+\frac{1}{2} \sum_{n=1}^{\infty} a_{n}\left(e^{2 n \pi i x}\right)+\frac{1}{2} \sum_{n=-\infty}^{-1} a_{-n}\left(e^{2 n \pi i x}\right) .
$$

\footnotetext{
${ }^{3}$ son las fórmulas para calcular los coeficientes de la series de Fourier.
} 
Definiendo

$$
\alpha_{n}=\left\{\begin{array}{lll}
\frac{a_{n}}{2} & \text { si } & n \geq 1 \\
\frac{a_{-n}}{2} & \text { si } & n \leq-1
\end{array}\right.
$$

se verifica que

$$
\alpha_{n}=\frac{(-1)^{n}-1}{2 n^{2} \pi^{2}} .
$$

Luego, usando la ecuación (29), la ecuación (26), se puede escribir como:

$$
\begin{aligned}
|x| & =\frac{1}{4}+\frac{1}{2} \sum_{n=1}^{\infty} a_{n} e^{2 n \pi i x}+\frac{1}{2} \sum_{n=-\infty}^{-1} a_{-n} e^{2 n \pi i x} \\
& =\frac{1}{4}+\sum_{n=1}^{\infty} \alpha_{n} e^{2 n \pi i x}+\sum_{n=-\infty}^{-1} \alpha_{n} e^{2 n \pi i x}=\frac{1}{4}+\sum_{n \in \mathbb{Z}-\{0\}} \alpha_{n} e^{2 n \pi i x} .
\end{aligned}
$$

Si se define $\alpha_{0}=\frac{1}{4}$, entonces se tiene

$$
|x|=\sum_{n \in \mathbb{Z}} \alpha_{n} e^{2 n \pi i x} .
$$

Luego, si se sustituye $x=r h$ con $h \neq 0$ en la ecuación (30), resulta

$$
|r|=\frac{1}{h} \sum_{n=-\infty}^{\infty} \alpha_{n} e^{2 n \pi i r h}, \text { con } \alpha_{0}=\frac{1}{4} y \alpha_{n}=\frac{(-1)^{n}-1}{2 n^{2} \pi^{2}} .
$$

Como $x \in[-1 / 2,1 / 2]$ entonces $r \in I=[-1 / 2 h, 1 / 2 h]$, teniendo en cuenta que si $h$ es muy pequeño, entonces $I$ se parece a $(-\infty, \infty)$, entonces la ecuación $(24)$, se cumple que

$$
Q_{\theta}(s) \approx \int_{I}|r| \widehat{\mathcal{P}_{\theta} f}(r) e^{2 \pi i r s} d r
$$

Luego, usando las ecuaciones (31) y (32), se obtiene

$$
\begin{aligned}
Q_{\theta}(s) & \approx \int_{I}|r| \widehat{\mathcal{P}_{\theta} f}(r) e^{2 \pi i r s} d r=\frac{1}{h} \sum_{n=-\infty}^{\infty} \alpha_{n} \int_{I} \widehat{\mathcal{P}_{\theta} f}(r) e^{2 \pi i r s}\left(e^{2 n \pi i r h}\right) d r \\
& =\frac{1}{h} \sum_{n=-\infty}^{\infty} \alpha_{n} \int_{I} \widehat{\mathcal{P}_{\theta} f}(r) e^{2 \pi i r s+2 n \pi i r h} d r=\frac{1}{h} \sum_{n=-\infty}^{\infty} \alpha_{n} \int_{I} \widehat{\mathcal{P}_{\theta} f}(r) e^{2 \pi i(s+n h) r} d r
\end{aligned}
$$

Si esta última integral lo extendemos a $\mathbb{R}$ tenemos:

$$
Q_{\theta}(s) \approx \frac{1}{h} \sum_{n=-\infty}^{\infty} \alpha_{n} \int_{-\infty}^{\infty} \widehat{\mathcal{P}_{\theta} f}(r) e^{2 \pi i(s+n h) r} d r
$$

Ahora esta última integral es la transformada inversa de $\widehat{\mathcal{P}_{\theta} f}(r)$, por lo tanto:

$$
Q_{\theta}(s) \approx \frac{1}{h} \sum_{n=-\infty}^{\infty} \alpha_{n} \mathcal{P}_{\theta} f(s+n h)
$$

La ecuación (33) no tiene transformadas de Fourier, es simplemente una sumatoria y que de hecho es finita porque $\mathcal{P}_{\theta} f$ es de soporte compacto, pues la proyección ortogonal de un compacto es un compacto. [3], [10], $[8],[14]$ 


\subsection{Aplicación: Modelo de Shepp-Logan ("Head Phantom")}

El modelo de Shepp-Logan es presentado como un modelo básico para el análisis de la precisión de los métodos de reconstrución presentados en esta sección.

Como citado por Slaney [8], las del cerebro pueden ser considerados las imágenes que exigen más precisión, flexibilidad y complejidad de un algoritmo de reconstrucción. El modelo de un cerebro fue idealizado por Shepp-Logan, y es frecuentemente utilizado para comprobar la precisión de los algoritmos de reconstrucción. Este modelo está basado en un conjunto de 10 elipses.

El término "phantom" en Matlab, genera una imagen del espectro de la cabeza. Una ventaja de usar una imagen como la del modelo de Shepp-Logan para simulaciones en computador es que se puede usar expresiones analíticas para la proyección usando transformada de Radon (Tomado de [8]). La proyección de la imagen compuesta por un número de elipses es simplemente la suma de las proyecciones de cada una de las elipses. Esto es debido a la linearidad de la transformada de Radon.

En esta parte se usarán las funciones internas de Matlab para generar una imagen patrón y luego trataremos de reconstruirla usando diferente número de proyecciones. Con el programa (5.1), se genera la figura 7 .

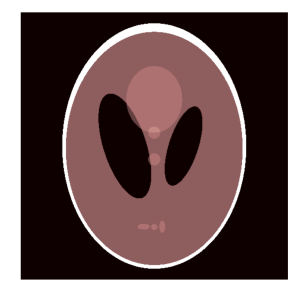

Figura 7: Imagen patrón de la cabeza.

Fuente. Chávez (2009, p. 72)

La figura 7, consta de una elipse grande (representando el cerebro) conteniendo varias elipses más pequeñas (representando características en el cerebro).Ahora se va a a reconstruira a la imagen patrón y veamos la exactitud numérica de la reconstrucción, para ello se usa los algoritmos ya diseñados e implementados en matlab como RADON e IRADON ${ }^{4}$

Con el programa (5.2) se obtienen las imágenes que se muestran en la figura 8

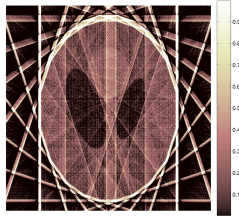

(a) 10 proyecciones

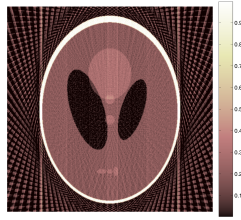

(b) 50 proyecciones

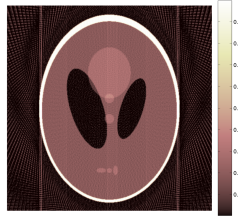

(c) 100 proyecciones

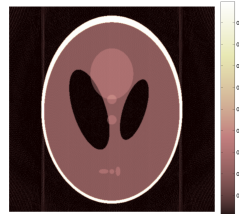

(d) 250 proyecciones

Figura 8: Proyecciones.

Fuente. Chávez (2009, págs. 77 y 75)

\footnotetext{
${ }^{4}$ Funciones internas de Matlab
} 


\section{Experimento: Estructura interna de tubos}

El objetivo de esta sección es el de explicar las nociones de la fórmula de reconstrucción usando proyecciones. Como no se cuenta con una máquina de rayos X, para verificar si en realidad podemos recuperar la función $f$ usando la transformada de Radon $\mathcal{P}_{\theta} f$, consideraremos secciones muy particulares que tengan con simetría radial. De esa manera nosotros mismos podemos hallar la proyección ${ }^{5}$ manualmente. Para tener una idea concreta, imaginemos que tenemos unos tubos con simetría radial acotados por $S^{1}(\mathbb{R})$ y deseamos saber, sin romperlos, cómo son internamente; es decir, si los tubos son compactos, si tienen una parte hueca, o si tienen un alma (zona central) de mayor densidad. Consideremos tres tubos que respondan cada uno a una de estas características: Uno compacto de radio 1 y densidad 1, otro igual que el anterior pero con la zona central $0 \leq r \leq 1 / 2$ hueca, y un tercero con esta zona central rellena de un material de densidad 2, como se muestra en la figura 9 .

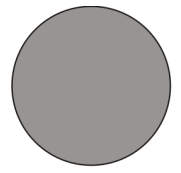

Tubo relleno

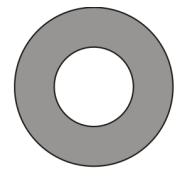

Tubo hueco

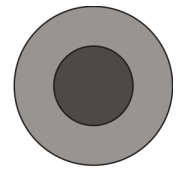

Tubo con alma

Figura 9: Tipos de tubos.

Fuente. Chávez (2009, p. 82)

Trabajar con este tipo de estructuras (los tubos) facilita hallar las proyecciones pues tienen simetría radial, entonces la función proyección ("sombra") $S(t)=\mathcal{P}_{\theta}(t)$ no dependerá del ángulo $\theta$ y se puede calcular fácilmente, es decir, si se hiciera el experimento con rayos X, se puede deducir las proyecciones son:

Para el tubo relleno.

$$
\mathcal{P}_{\theta} f(x)=S_{1}(x)=\left\{\begin{array}{lll}
2 \sqrt{1-x^{2}} & \text { si } & |x| \leq 1 \\
0 & \text { si } & |x|>1
\end{array}\right.
$$

Para el tubo con interior hueco.

$$
S_{2}(x)=S_{1}(x)-\frac{1}{2} S_{1}(2 x) .
$$

Para el tubo con interior con parte central rellena de un material con mayor densidad.

$$
S_{3}(x)=S_{1}(x)+\frac{1}{2} S_{1}(2 x) .
$$

Si se usa el programa (5.3) se obtiene la gráficas de las funciones sombras (Ver figura 10).

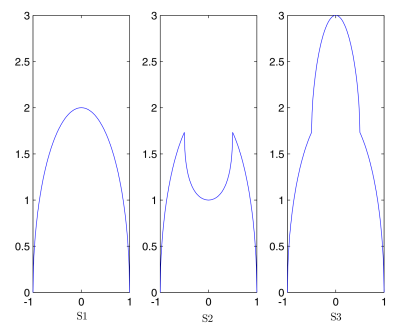

Figura 10: Gráficas de las funciones sombra.

Fuente. Chávez (2009, p. 83)

\footnotetext{
${ }^{5}$ algunos autores lo llaman sombras
} 
El experimento consiste en que haciendo trabajar la calculadora o una computadora, se puede recuperar la estructura del tubo a partir de la función $S_{i}$.

Entonces para resolverlo, según lo visto en la sección 3.1, es necesario aproximar la ecuaciones (23) y (33). La ecuación (33) se puede aproximar usando la regla del trapecio y tomando proyecciones a lo largo del eje $X$, resulta la ecuación (34), (más detalles ver [1, sección 4.3])

$$
f(R, 0) \approx h \sum_{\theta_{j}} Q_{\theta_{j}}\left(R \cos \theta_{j}\right)
$$

donde $\operatorname{los} \theta_{j}$ recorren $[0, \pi]$ de $h$ en $h$, es decir, $\theta_{0}=0, \theta_{1}=h, \theta_{2}=2 h$ y si sucesivamente.

Por otro lado teniendo en cuenta las simetrías $\alpha_{n}=\alpha_{-n}$ y de $S_{i}(-u)=S_{i}(u)$, para $i=1,2,3$. y puesto que en nuestro caso $\mathcal{P}_{\theta_{j}} f=S_{i}$ se tiene que la ecuación (33), se transforma en

$$
Q_{\theta_{j}}(s) \approx \frac{1}{h}\left(\frac{S_{i}(s)}{4}+2 \sum_{n=1}^{\infty} \alpha_{n} S_{i}(s+n h)\right) .
$$

De forma concreta, lo que se tiene que hacer es fijar una precisión $h$ pequeña (aunque si es demasiado pequeña el programa demorará algunos minutos) y hacer un programa que permita calcular para cada coordenada radial $R$, las ecuaciones (34) y (35).

La exactitud que se logra al aproximar la densidad en $\|\vec{x}\|=R$ por $f(R, 0)$ es como se esperaba. Por ejemplo, tomando $\mathrm{h}=0.001$ y usando los programas (5.4), se obtiene la reconstrucción del tubo 1 , haciendo pequeñas modificaciones a la función sombra se pueden obtener un programa para recontruir el tubo 2 y el tubo 3 (mas detalles ver [4]).

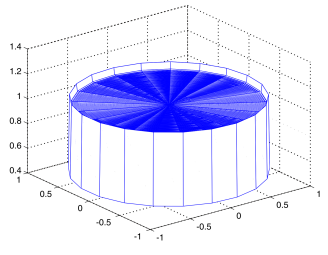

(a) tubo 1

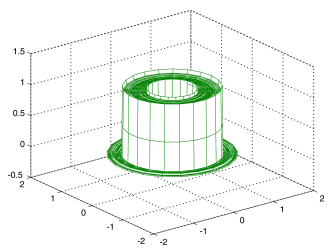

(b) tubo 2

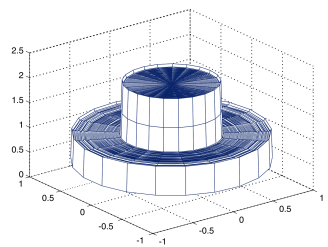

(c) tubo 3

Figura 11: Reconstrucción de los tubos. Fuente. Chávez (2009, págs. 74, 75 y 76)

\section{Conclusiones}

- Es posible utilizar el teorema de las "Rebanadas" para reconstruir la estructura interna de los objetos, sin necesidad de abrirlos.

- En el experimento de los tubos podemos observar que utilizando el teorema de las rebanadas y la Transformada de Fourier Discreta es posible reconstruir computacionalmente las funciones "sombras".

- En [8, Sección 3] existen otras fórmulas de reconstrucción para $y=\mathcal{K} x$

- Una forma interesante de resolver este problema, sería usando elementos finitos.

- Una variante sería tomar proyecciones en otra forma como por ejemplo en forma de abanico (ver figura $12)$. 


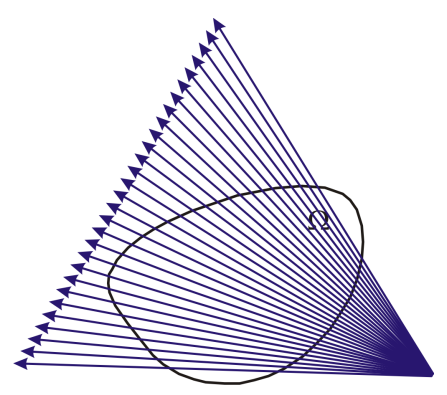

Figura 12: Proyección en forma de abanico. Fuente. Chávez (2009, p. 76)

\section{Referencias bibliográficas}

[1] Burden, R., Faires, J., Burden, A. (2011). Numerical Analysis. USA: Cengage Learning .

[2] Carbajal, E. (1999). Problemas inversos y sus aplicaciones. Lima: Serie A:Monografías, Colección de Matemáticos del Perú.

[3] Chamizo, F. (2003). Notas del curso Modelización II. Recuperado el 15 de abril del 2008 de http://www.uam.es/personal_pdi/ciencias/fchamizo/libreria/fich/APmodII03.pdf

[4] Chávez, E. (2009). Solución al Problema Inverso Tomografía Computarizada Tratamiento Numérico. Tesis de Licenciatura en Matemática Pura. Universidad Nacional Mayor de San Marcos, Perú.

[5] Dym, H., McKean, H. (1972). Fourier Series and Integrals. New York: Academic Press.

[6] Groetsch, W. (1999). Inverse Problems: activities for undergraduates. Washington, DC: Mathematical Association of America. .

[7] Hansen, C. (1998). Rank-Deficient and Discrete Ill-posed Problems. Philadelphia: Society for Industrial and Applied Mathematics.

[8] Avinash, K., Malcolm, S. (1998). Principles of Computerized Tomographic Imaging. New York: SIAM, IEEE. Press.

[9] Kirsch, A. (1996). An Introduction to the Mathematical Theory of Inverse Problems. New York: SpringerVerlag

[10] Natterer, F. (2013). The Mathematics of Computerized tomography. New York: Wiley.

[11] Quarin (s.f). Tomografía por rayos X. Recuperado el 12 de noviembre del 2007 de www.geocities.com/tomografiademadeira/autor2.html.

[12] Ramm, G., Katsevich, I. (1996). The Radon Transform and Local Tomography. Florida: CRC Press. Boca Raton.

[13] Kennan, S., Donald, S., Sheldon, W. (1977). Practical and mathematical aspects of the problem of reconstructing objects from radiographs. Bulletin of the American Mathematical. Society, 83, (6), 1227 $-1270$.

[14] Taylor, E. (1996). Partial Differential Equations I. New York: Springer. 


\section{Anexo}

\section{Programas para la sección 3.2}

\section{Programa 5.1}

Tipo:Matlab

Descripción: Genera una imagen patrón de la cabeza, con un color determinado por el comando pink.

$\% \%$ EL COMANDO PHANTOM GENERA UNA IMAGEN PATRÓN

$\mathrm{X}=$ phantom (512);

$\% \%$ GENERA UNA VENTANA DE FIGURA

imshow (X);

$\% \%$ GENERA EL COLOR EN LA IMAGEN

colormap (pink);

\section{Programa 5.2}

Tipo:Matlab

Descripción: Permite generar una imagen usando $n$ proyecciones con un color determinado por el comando pink.

\section{$\% \%$ RECONSTRUCCIÓN USANDO N PROYECCIONES}

$\mathrm{n}=$ input ('ingresa el número de proyecciones: $\mathrm{n}=$ ') ;

$\mathrm{h}=180 / \mathrm{n}$;

theta $=[0: h: 180]$;

$[R, a]=$ radon $(X$, theta $)$;

$\% \%$ GENERA UNA VENTANA DE FIGURA

imshow (iradon $(\mathrm{R}, \mathrm{h})$ )

$\% \%$ GENERA EL COLOR EN LA IMAGEN CON LA BARRA DE COLORES $\% \% \%$

colormap (pink); colorbar;

\section{Programas para la estructura interna de tubos}

\section{Programa 5.3}

Tipo:Matlab

Descripción: Generar las gráficas de las sombras de las proyecciones de los tubos.

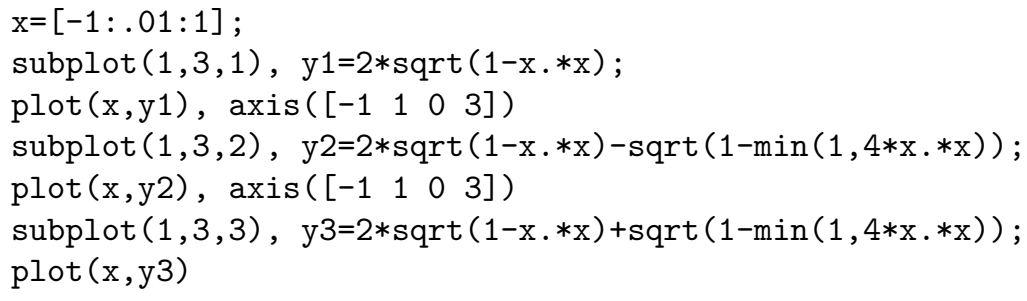




\section{Programa 5.4}

Tipo:Matlab

Descripción: Da las aproximaciones para reconstruir la estructura interna del tubo 1 con su gráfica.

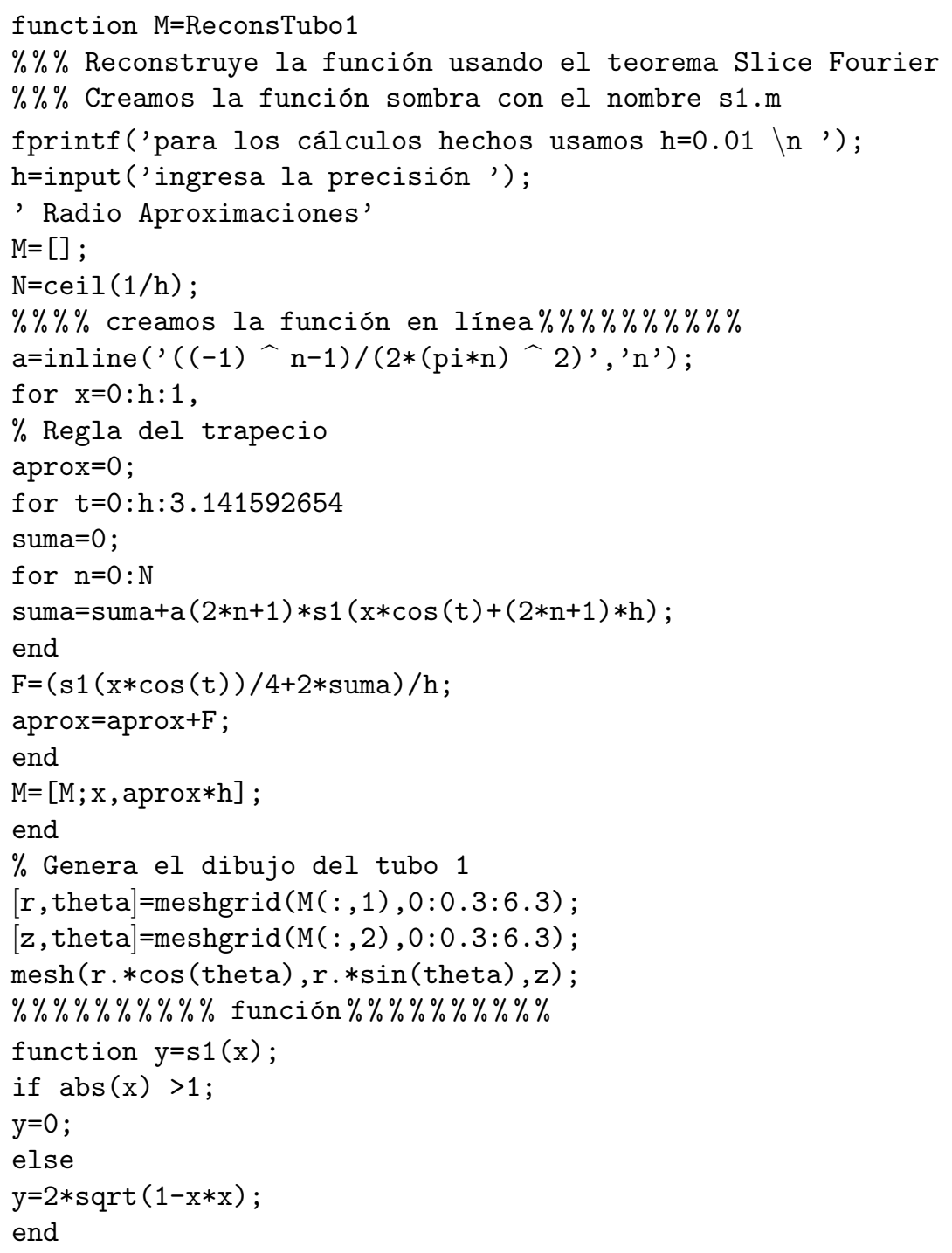

